\title{
Integration of commercial screen-printed electrodes into a voltammetric electronic tongue for the analysis of aminothiols
}

\author{
Ricardo H. P. Pedroza ${ }^{a}$, Núria Serrano ${ }^{b}$, José Manuel Díaz-Cruz ${ }^{b} *$,Cristina Ariño ${ }^{b}$, Miquel Esteban ${ }^{b}$ \\ a Biological Chemistry and Chemometrics, Institute of Chemistry, Federal University of Rio Grande do Norte, 59072-970, Natal- \\ RN, (Brazil). \\ b Departament de Química Analítica, Facultat de Química, Universitat de Barcelona, Martí i Franquès 1-11, E-08028 - \\ Barcelona (Spain). \\ * Phone: (34) 9340391 16. Fax: (34) 934021233. \\ e-mail: josemanuel.diaz@ub.edu
}

Received: ((will be filled in by the editorial sttaff))

Accepted: ((will be filled in by the editorial sttaff))

\begin{abstract}
A voltammetric sensor array (or electronic tongue) is developed for the simultaneous quantification of cysteine, glutathione and homocysteine without need of previous separation. It is based on the integration of three commercial screen-printed electrodes (gold curated at high and low temperature and carbon modified with carbon nanotubes). Linear sweep voltammograms measured simultaneously by all three sensors are processed by Partial Least Squares (PLS) regression and different variables selection algorithms such as Genetic Algorithm and interval-Partial Least Squares. The method was applied to synthetic mixtures and successfully validated, with correlation coefficients of prediction $\left(\mathrm{R}_{\mathrm{p}}{ }^{2}\right)$ of $0.9542,0.9429$ and 0.9589 for cysteine, glutathione, and homocysteine respectively.
\end{abstract}

Keywords: voltammetric electronic tongue, screen-printed electrodes, aminothiols, partial least squares calibration.

DOI: 10.1002/elan.((will be filled in by the editorial sttaff))

\section{Introduction}

Thiol groups play a crucial role in biological systems. Cysteine (Cys), homocysteine (hCys) and glutathione (GSH) are among the most studied thiol-containing peptides, since they are involved in important processes of metabolism and homeostasis [1]. Different levels of these thiols in biological systems can be an indication of some serious illnesses. For instance, high levels of hCys in plasma are strongly correlated with an increased risk of coronary artery disease and cerebrovascular disease [2, 3]. As for GSH, it is the main non-enzymatic component of the antioxidant system in organisms [4]. Thus, monitoring thiol levels in biological fluids is an important task within the biomedical community.

Various methods have been reported for the analysis of thiols. Among these, liquid chromatography with UV or fluorescence detection plays a key role. However, such chromatographic methods usually require derivatization to introduce chromophore or fluorophore groups [5]. In contrast, electrochemical methods could take advantage of the electroactive character of thiol groups (susceptible to be oxidized to disulphide forms) to develop simple, rapid, low cost and easily automated screening assays without need of derivatization.

This is why many kinds of electrodes have been tested for thiol detection in biological and environmental samples [6-8]. However, conventional electrode materials produce a quite slow oxidation of thiol compounds, which results in a poor sensitivity. A special situation is produced when using mercury or mercury-gold amalgam electrodes. Then, the (quite fast) oxidation detected is not that of thiol groups, but the oxidation of mercury to be complexed by them [9]. Besides mercury, new materials have shown interesting capabilities for the oxidation of thiol molecules. This is the case of the electrocatalytic properties of edge-plane pyrolytic graphite [10] or the extended potential domain provided by boron doped diamond [11]. Recently, silver electrodes have been successfully applied to the amperometric detection of thiols in liquid chromatography [12,13]. Although chemisorption of thiols on silver by formation of selfassembled monolayers [14] produce similar surface fouling problems as in gold electrodes, silver electrodes can be electrochemically cleaned on-line by means of a pulsed potential waveform. 
Modification of conventional solid electrodes with mediators can considerably increase the electrochemical oxidation rate of thiols, thus improving their detection. Among the mediators used, we can mention vanadium and ruthenium compounds [15], polymeric cobalt phthalocyanine [16] and, especially, nanoparticles [17-20] mostly based on gold and multi-walled carbon nanotubes (MWCNT).

Recently, disposable screen-printed electrodes (SPE) have opened a promising field of research in electrochemical analysis, especially since they are commercially available in a large variety of materials and designs, with many possibilities of modification [21-25]. The main advantages of these devices are their low cost, potential portability, simplicity of operation, and the compact arrangement containing the working, auxiliary and reference electrodes. Their low cost allows using them as disposable electrodes, thus avoiding time-consuming cleaning procedures to minimize the effects of electrode fouling and contamination. Nevertheless, studies using SPE for detecting thiols are still scarce. For example, it has been reported the use of SPE modified with a polymer film [26] or carbon SPE modified with $\mathrm{MnO}_{2}$ particles [27,28]. Also, the different reaction rates of hCys and GSH with electrochemically oxidized catechol on carbon nanotubes SPE have been used to determine both peptides [29]. As for reverse-phase liquid chromatography, commercial gold SPE have been successfully applied to the amperometric detection of several aminothiols in plasma samples [30].

In general, these electrochemical methods are poorly selective and, unless a chromatographic separation is included, they are only applicable to total thiol determination or to the resolution of relatively simple mixtures of thiol molecules. It is true that chemometric methods like partial least squares calibration (PLS) or multivariate curve resolution by alternating least squares (MCR-ALS) have been successfully used for the analysis of unresolved signals obtained by linear sweep voltammetry and other electroanalytical techniques [3136]. However, in the case of different thiol molecules, their electrochemical behaviour is so dependent on the thiol group that their signals are very similar with each other [37]. This usually produces a strong overlapping which hinders the resolution of complex thiol mixtures without a previous separation step.

An intermediate way between the chromatographic analysis of intricate thiol-containing samples and the direct sensing of a target thiol molecule in the absence of notorious interferences could be the use of a voltammetric sensor array. Sensor arrays, also known as electronic tongues, consist of the combination of several non-specific sensors to obtain multivariate data sets that can be further analyzed by chemometric methods in order to characterize liquid samples or determine some of their components. Although electronic tongues are mainly used in food analysis as tasting devices, they are also useful in other research fields such as environmental analysis or bioanalysis [38-42].

The aim of this work is to carry out the preliminary design of a voltammetric tongue for the analysis of aminothiols, entirely based on commercially available SPE. After a previous test of different electrode materials, two kinds of gold-SPE (cured at different temperatures) and a carbon-SPE modified with multi-walled carbon nanotubes (MWCNT) have been selected to do simultaneous linear sweep voltammetric (LSV) measurements in a multichannel potentiostat on different synthetic mixtures of Cys, hCys and GSH. The application of principal component analysis (PCA) and partial least squares (PLS) allowed us to test the capabilities of the electronic tongue for sample characterization and thiol quantification.

\section{Experimental}

\subsection{Chemicals}

All reagents were analytical grade. L-cysteine (Cys, 97\%), DL-homocysteine (hCys $\geq 95 \%$ ) and trifluoroacetic acid (TFA 99\%) were purchased by Sigma-Aldrich (Sta. Louis, USA). Reduced glutathione (GSH > 98\%), and N,Ndimethylformamide (DMF) were obtained by Merck (Darmstadt, Germany). Multi-walled carbon nanotubes (MWCNT) solution was purchased by DropSens (Oviedo, Spain, ref. DRP-MWCNTCOOH). Potassium chloride was provided by Panreac (Barcelona, Spain). All samples were prepared using ultrapure filtered water obtained by Milli-Q plus 185 purification system (Merck Millipore Corporation, Germany).

\subsection{Apparatus}

Voltammetric measurements were registered at room temperature $\left(20^{\circ} \mathrm{C}\right)$ with a $\mu$ Stat 8000 potentiometer DropSens (Oviedo, Spain) attached to several screenprinted devices by means of a multichannel cable DRPCABSTATMULTI Dropsens and a DRP-CAC cable for each screen-printed unit. The multichannel cable was also connected to an external $\mathrm{Ag} / \mathrm{AgCl}$ reference electrode Metrohm (Herisau, Switzerland), to which all potentials are referred, and to an external platinum auxiliary electrode, also by Metrohm. In this way, the potentiometer could simultaneously measure the signals of the working electrodes of all screen-printed units referred to a common reference electrode and a common auxiliary electrode. A glass cell and a cell support, both by Metrohm, were used to contain the cell solution and up to five electrodes (usually, the reference electrode, the auxiliary electrode and three screen-printed electrodes). A magnetic stirrer by 
IKA (Staufen, Germany) and purified nitrogen were also available for stirring and deaeration of the solutions. Figure 1 illustrates the above-mentioned experimental setup.

Three types of commercial screen-printed devices were used, all of them by Dropsens. They mainly differ in the working electrode, which is made of gold with high temperature curing (ref. 220-AT), gold with low temperature curing (ref. 220-BT) or carbon (ref. 110). The units also include an auxiliary electrode (made of the same material as the working) and a silver pseudo-reference electrode printed on an alumina substrate. An insulating layer delimits the working area and protects the electric contacts. In multichannel mode, the pseudo-reference and auxiliary electrodes of the screen-printed devices are not used during the measurements, being substituted by the common external reference and auxiliary electrodes.

Linear sweep voltammograms (LSV) were recorded in triplicate (i.e., three successive scans with the same screenprinted device) using the range $0.1-0.9 \mathrm{~V}$ with a potential step of $0.002 \mathrm{~V}$ and a scan rate of $0.05 \mathrm{Vs}^{-1}$. Data acquisition was made through DropView software by Dropsens and home-made programs implemented in Matlab® environment [43].

A mixer MixMate PCB-08 by Eppendorf (Hamburg, Germany), and an ultrasonic bath Bransonic model 2510EMTH provided by Branson Ultrasonics (Danbury, USA) were used for SPE modification with carbon nanotubes.

\subsection{Experimental procedures}

\subsubsection{Modification of carbon screen-printed electrodes with carbon nanotubes}

The modification of carbon SPE with carboxyl modified multiwalled carbon nanotubes (DRP-MWCNTCOOH) has been described elsewhere [44]. According to this procedure, $1 \mathrm{mg}$ of carbon nanotubes was mixed with 1 $\mathrm{mL}$ of DMF during 12h at $2000 \mathrm{rpm}$ using Eppendorf Mixmate and later in an ultrasonic bath during $1 \mathrm{~h}$. Then, a dilution is made with DMF: $\mathrm{H}_{2} \mathrm{O}$ (1:1) until a concentration of $0.5 \mathrm{mg} \mathrm{mL} \mathrm{m}^{-1}$. Finally, $4 \mu \mathrm{L}$ of such dispersion was pipetted on the working electrode surface of the carbon SPE and dried at room temperature.

\subsubsection{Preparation of solutions for PLS calibration}

Synthetic samples containing different proportions of Cys, GSH and hCys were used for constructing PLS calibration models. All samples were prepared with ultrapure water, $0.05 \% \mathrm{TFA}$ and $\mathrm{KCl} 10^{-3} \mathrm{~mol} \mathrm{~L}^{-1}$. The concentration of each peptide was at four levels in binary mixtures $(0$, $2 \times 10^{-5}, 4 \times 10^{-5}$ and $\left.6 \times 10^{-5} \mathrm{~mol} \mathrm{~L}^{-1}\right)$ and at three levels in ternary mixtures $\left(0,3.0 \times 10^{-5}\right.$ and $\left.6.0 \times 10^{-5} \mathrm{~mol} \mathrm{~L}^{-1}\right)$, according to the experimental designs shown in Figure 2. The values of the concentrations were chosen to be clearly inside the linearity ranges commonly found in our previous works with these substances and electrodes under similar conditions (e.g. ref. [30]).

\subsubsection{Data analysis}

Data matrices for every individual sensor were built from the linear sweep voltammograms measured for all samples, so that every element in the row $i$ and the column $j$ contains the current measured in $\mu \mathrm{A}$ for the sample $i$ at the potential $j$. As measurements were made in triplicate, the voltammogram in every row was indeed the mean of the currents obtained in all three scans. The relative standard deviation of such replicates ranged between 1 and $4 \%$ of the overall current, depending on the solution and the kind of electrode.

When different sensors measure together in the same sample (sensor array mode) the overall data matrix is made by row-wise augmentation of the individual sensor matrices. In principle, all currents are included in multivariate analysis, unless a variable selection method is applied to reduce the size of the dataset.

Pre-processing of the data matrices, variable selection, principal component analysis (PCA) and construction of the partial least squares (PLS) model were made using Matlab® [43] with PLS-toolbox [45]. Different preprocessing techniques were evaluated, including mean center, baseline correction, multiplicative scattering correction (MSC), variance scaling, smoothing SavitzkyGolay, and 1st and 2nd derivative Savitzky-Golay.

The accuracy of PLS calibration has been evaluated by means of the square correlation coefficient, $\mathrm{R}^{2}$, computed from the line fitted to the predicted versus experimental concentration plot inside the calibration $\left(\mathrm{R}_{\mathrm{C}}{ }^{2}\right)$ or validation $\left(\mathrm{R}_{\mathrm{P}}{ }^{2}\right)$ sets. Additionally, the corresponding mean root square errors (RMSE) have been considered, computed as:

$$
\text { RMSE }=\sqrt{\frac{\sum_{i=1}^{n}\left(y_{i}-\widehat{y}_{i}\right)^{2}}{n}}
$$

where $\boldsymbol{y}_{\boldsymbol{i}}, \hat{\boldsymbol{y}}_{\boldsymbol{i}}$ are the experimental and the predicted concentration values and $\mathrm{n}$ is the number of samples used for calibration (RMSEC) or prediction (RMSEP). 

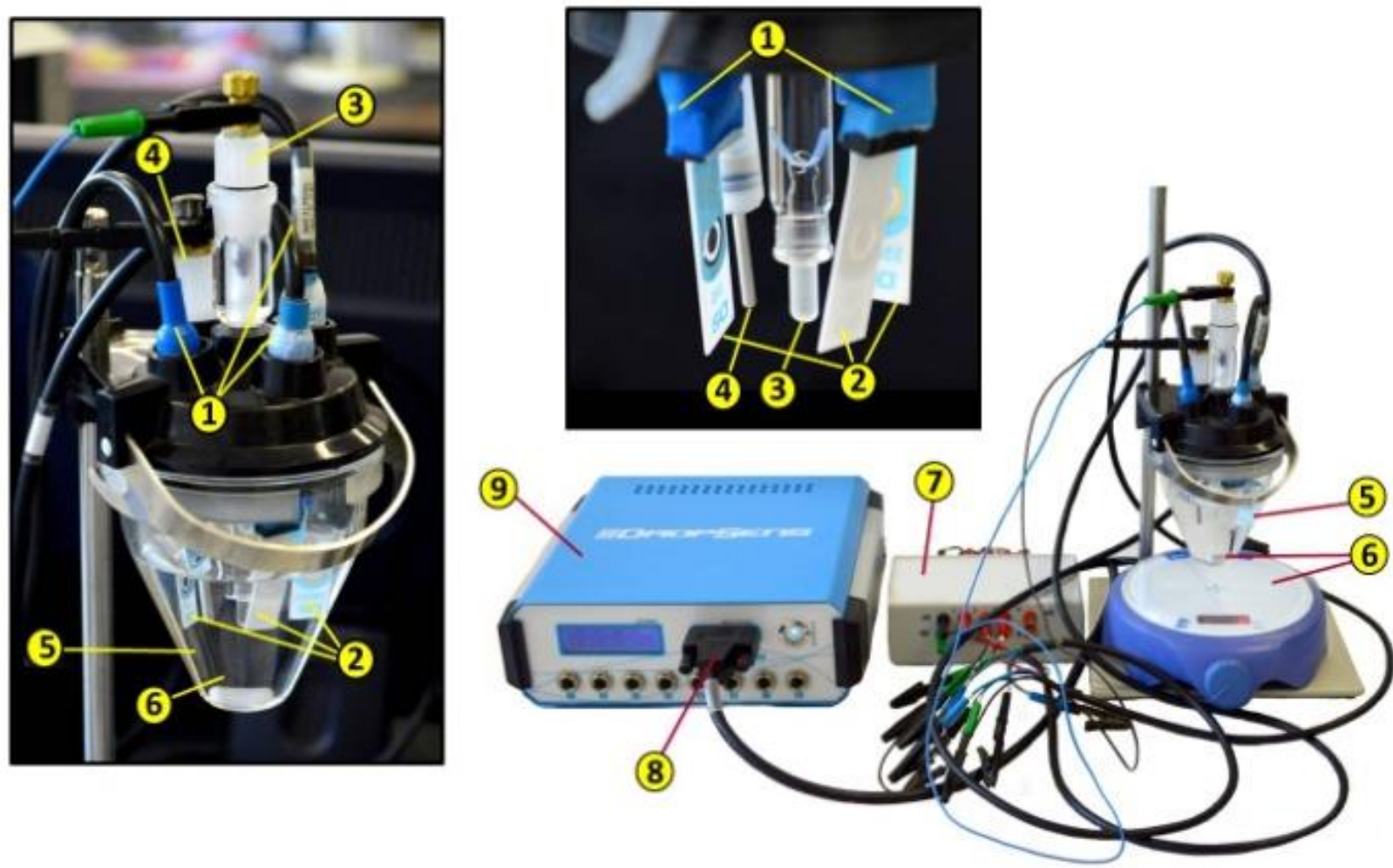

Fig. 1. Experimental setup for simultaneous LSV measurements with three different screen-printed electrodes. (1. DRP-CAC connectors. 2. Screen-printed electrodes. 3. Ag/AgCl reference electrode. 4. Pt auxiliary electrode. 5. Voltammetric cell. 6. Magnetic stirrer. 7. Connectors box. 8. Multichannel cable DRP-CABSTATMULTI. 9. Multichannel potentiostat $\mu$ Stat 8000).

a)

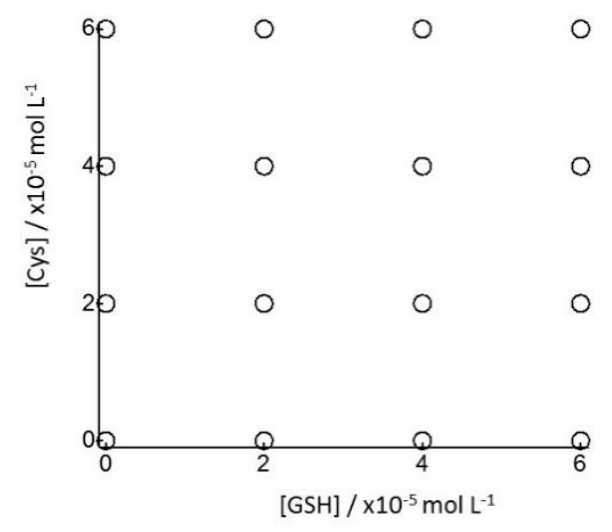

b)

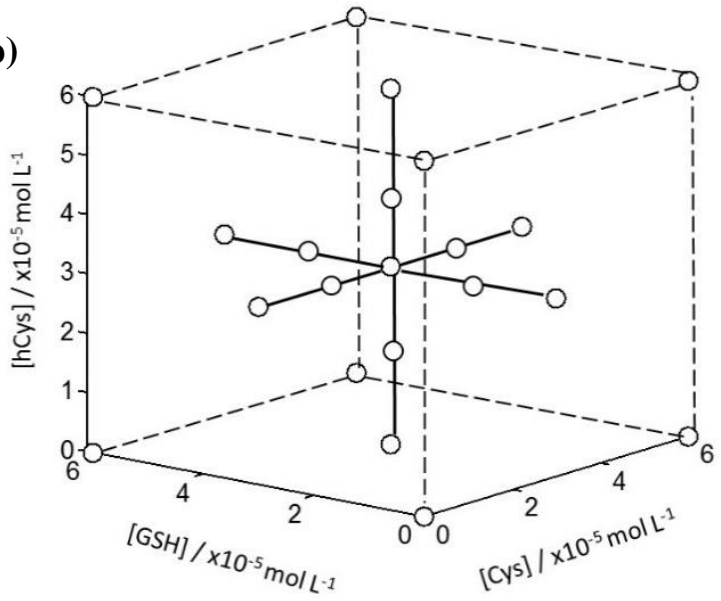

Fig. 2. Concentrations of Cys, GSH and hCys in the solutions used to build two- (a) and three-analytes (b) PLS models. Some of the solutions in (b) are used to build the model and the rest are considered in the further validation, according to Kennard-Stone selection algorithm.

\section{Results and discussion}

\subsection{Preliminary tests}

LSV signals were obtained in triplicate separately for five solutions of Cys and GSH by using four types of screen-printed devices: high temperature gold electrodes (AuAT), low temperature gold electrodes (AuBT), bare carbon electrodes and carbon electrodes modified with multi-walled carbon nanotubes (CNT). As Figure 3 shows, the voltammograms obtained with bare carbon electrodes exhibit too low currents, denoting a slow electrochemical oxidation of thiol groups. In contrast, the two types of gold electrodes and the CNT-modified carbon electrode produce higher currents, which suggest a faster oxidation kinetics. However, the comparison with background signals in the absence of thiols (Figure 3a) shows that in the case of CNT an important proportion of the current 
increase is not due to faradaic phenomena (i.e. thiol oxidation), but to the significant growing of capacitive currents. This means that the improvement of CNT as compared to bare carbon electrodes is clear but not as dramatic as it could seem at the first sight. An important feature of CNT electrodes is that they provide voltammograms quite different to these obtained with gold electrodes. This is confirmed by the PCA of the augmented matrix containing voltammograms of both Cys and GSH solutions measured with AuAT, AuBT and CNT electrodes.

a)
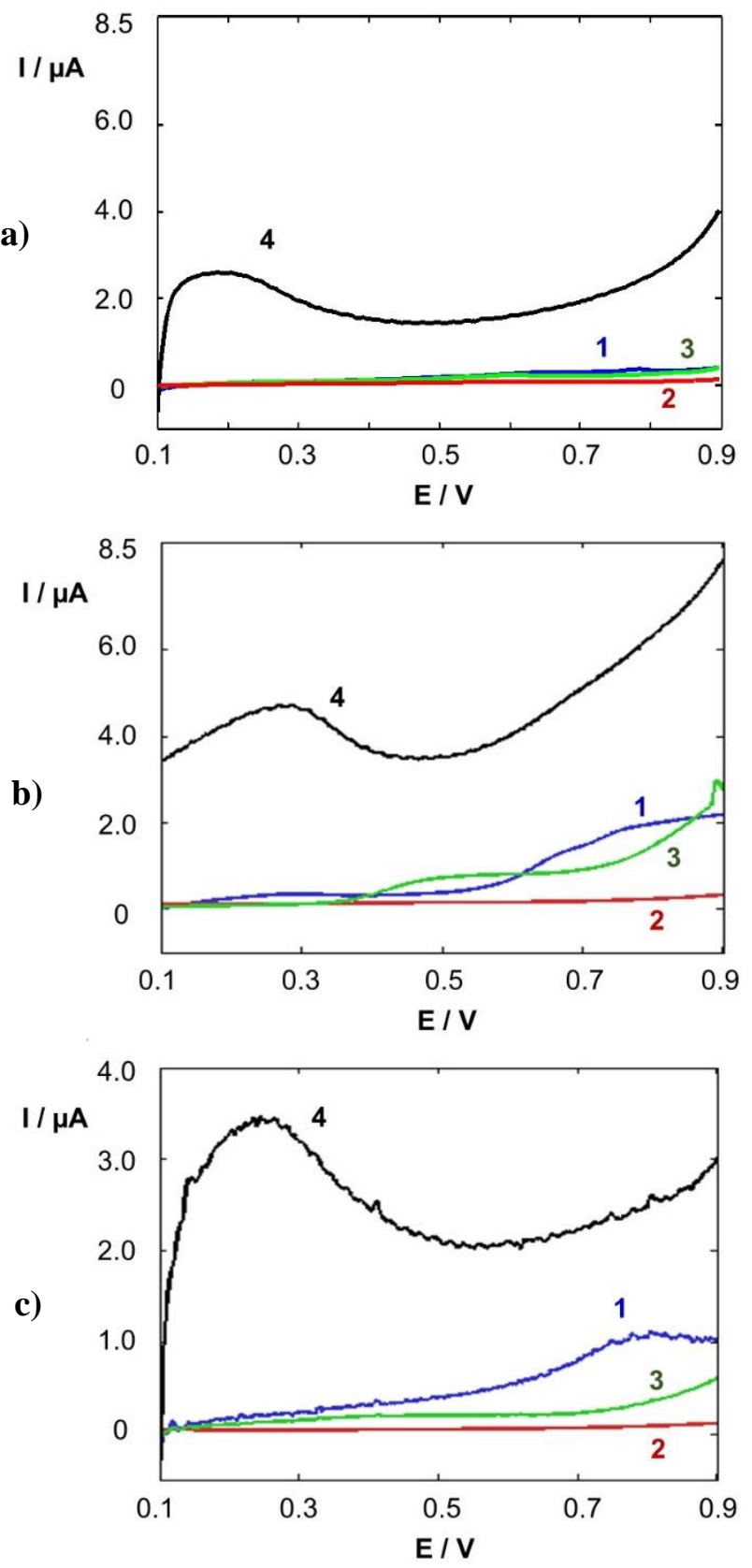

Fig. 3. LSV signals obtained with a blank (a) and with solutions $10^{-6} \mathrm{~mol} \mathrm{~L}^{-1}$ of Cys (b) and GSH (c) in a medium containing $0.05 \%$ of TFA and $10^{-3} \mathrm{~mol} \mathrm{~L}^{-1} \mathrm{KCl}$, measured with AuAT (1), carbon (2), AuBT (3) and CNT (4) screen-printed electrodes.
The PC2 vs. PC1 scores plot (Figure 4) shows that the combination of these three electrodes can difference quite well between Cys and GSH (the points corresponding to several replicates are very close with each other and far from the other groups, except for the Cys measurements with gold electrodes, whose points are slightly overlapping). Thus, the sensor array formed by AuAT, AuBT and CNT screen-printed electrodes is selected to carry out further experiments.

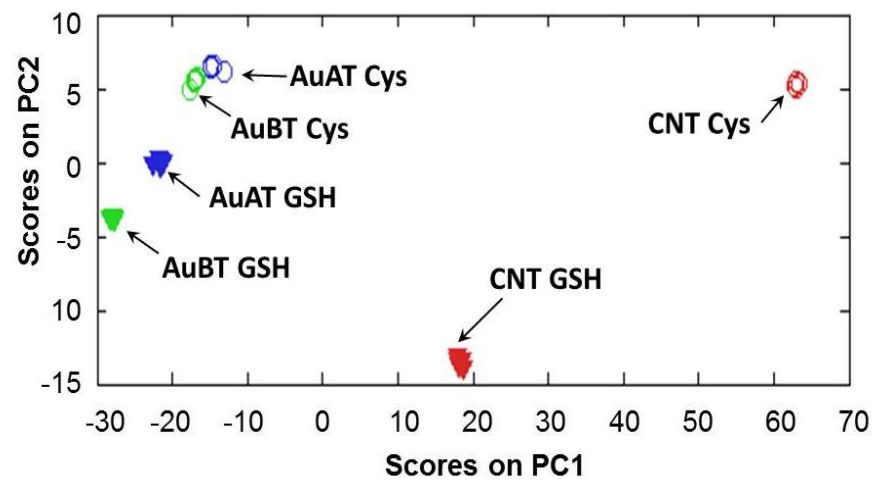

Fig. 4. PC2 vs. PC1 score plot of a data matrix containing LSV signals measured with AuAT, AuBT and CNT electrodes in five solutions containing Cys or GSH $10^{-6} \mathrm{~mol} \mathrm{~L}^{-1}$.

In order to test the calibration ability of the three-sensor array, a PLS model was made with solutions containing different proportions of Cys and GSH according to the experimental design in Figure 2a. Due to the complexity of the data (usually presenting potential shifts, scatter of signals or differences in the baseline which can affect the PLS performance), different types of data preprocessing were applied, such as baseline (BAS), smoothing Savitzky-Golay (SMOTH), multivariate scatter correction (MSC), variance (std) scalling (VARSTD), and mean center (MC). Variable selection algorithms (genetic algorithm-GA and interval partial least squares-iPLS) [4648 ] were also applied to select a minimum set of variables containing the maximum information related to the analytes concentration reducing the large number of variables obtained in this experiment.

The best model to quantify Cys was obtained using only AuAT electrode, with mean center (MC) as preprocess method, and three latent variables (LV). It was applied a variable selection by iPLS resulting in fifty selected variables which, upon GA selection, were reduced to eleven variables distributed in two potential regions: $0.25 \mathrm{~V}-0.35 \mathrm{~V}$ and close to $0.85 \mathrm{~V}$. In this way, the model presents a RMSECV of $0.7810^{-5} \mathrm{~mol} \mathrm{~L}^{-1}$ with an $\mathrm{R}_{\mathrm{CV}^{2}}$ of 0.8797 . In the case of GSH, the best model required the use of the augmented matrix with the three electrodes, MC as preprocess method and six latent variables. Variable selection was made by iPLS and resulted in ten ranges with five variables each, mostly in the regions $0.1-0.25 \mathrm{~V}$ and $0.7-0.85 \mathrm{~V}$. This model presents a RMSECV of $0.5610^{-5}$ mol L-1 with an $\mathrm{R}_{\mathrm{CV}^{2}}$ of 0.9366 . 


\subsection{Calibration and validation of PLS models in solutions containing all three analytes}

The preliminary test suggests that combining signals of the three electrodes could be useful not only to characterize and difference samples but also to determine some aminothiols in the presence of others. Then, by applying the knowledge extracted from the previous experience, a more complex calibration model was designed including three aminothiols: $\mathrm{Cys}$, GSH and hCys, a model which will be discussed in more detail.
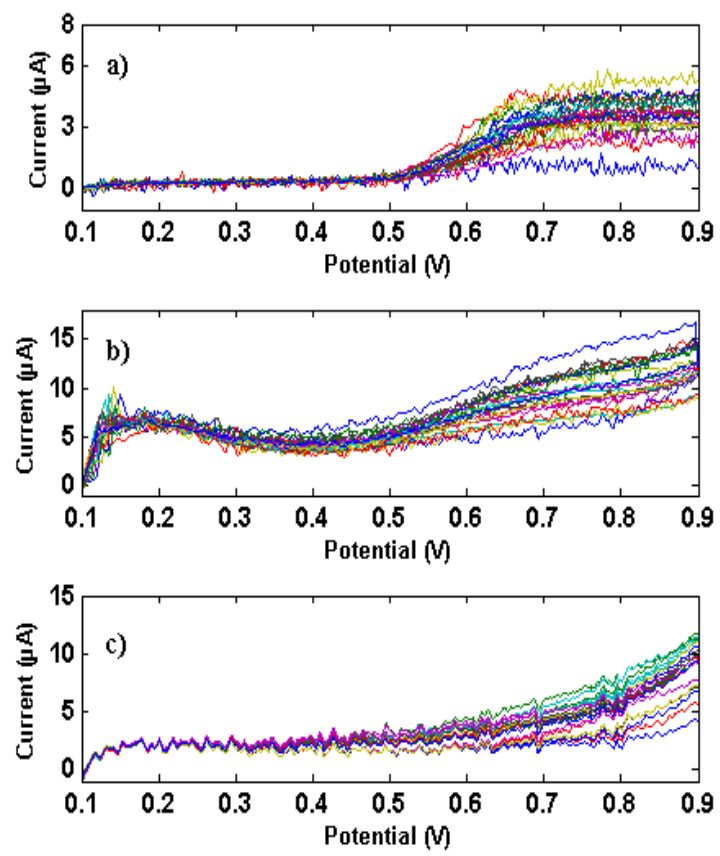

Fig. 5. LSV signals obtained by a) AuAT. b) CNT and c) AuBT electrodes in the calibration solutions whose composition is shown in Figure 2b.

Figure $2 \mathrm{~b}$ shows the composition of the 22 solutions and Figure 5 summarizes the LSV signals obtained with all three electrodes in all solutions. The solutions were divided into two groups applying the Kennard-Stone (KS) selection algorithm [49]. The groups consisted of fifteen and seven solutions for calibration and external validation, respectively. Variable selection was made in a similar way as in the previous model. Finally, Table 1 summarizes the main parameters of the best PLS calibration models attained for the quantification of each analyte.

In the case of Cys, the best results were obtained by using an augmented matrix with data from all three electrodes. Baseline correction and MC were applied as preprocess methods and variables were selected by GA. Then, a PLS model with seven latent variables yielded RMSEC, RMSEP values of $0.1110^{-5}$ and $0.5010^{-5} \mathrm{~mol} \mathrm{~L}^{-}$ ${ }^{1}$, respectively, and $\mathrm{R}^{2}$ of 0.9971 for calibration and 0.9542 for external validation.

The same augmentation, preprocess and variable selection strategies produced the best results for GSH quantification. A PLS model with six latent variables yielded RMSEC, RMSEP of $0.1110^{-5}$ and $0.5010^{-5} \mathrm{~mol}$ $\mathrm{L}^{-1}$, respectively, and $\mathrm{R}^{2}$ of 0.9973 for calibration and 0.9429 for external validation.

Data from two electrodes, MC preprocessing, variable selection by GA and iPLS and five latent variables were selected in the best PLS model to quantify hCys. It presents RMSEC, RMSEP of $0.9310^{-5}$ and $0.4910^{-5} \mathrm{~mol}$ $\mathrm{L}^{-1}$, respectively, and $\mathrm{R}^{2}$ of 0.8289 for calibration and 0.9589 for external validation.

Figure 6 shows the regression plot of predicted $v s$. measured concentrations for each analyte using the best models, being Figure $6 \mathrm{a}$ for Cys, Figure $6 \mathrm{~b}$ for glutathione, and Fig. 6c for hCys. In this experiment we have also compared the ideal values of intercept and slope $(0,1)$ with the estimated slope and intercept values obtained by elliptical joint confidence region (EJCR). If the ellipse contains the ideal point $(0,1)$, it means that there is no difference between true and predicted values in a 95\% confidence range [50]. Figure 7 represents the elliptical joint confidence region (EJCR) drawn for the best model of every analyte in this experiment. The plot confirms that both models were satisfactory with a good accuracy to simultaneously determine Cys, GSH and hCys. 
Table 1. Main parameters obtained in different PLS models applied to the determination of each analyte.

\begin{tabular}{lccccccc}
\hline $\begin{array}{l}\text { Model } \\
\text { Pretreatment }\end{array}$ & $\begin{array}{c}\text { Latent } \\
\text { Variables }\end{array}$ & $\begin{array}{c}\text { RMSEC } \\
\left(\times 10^{-5} \mathrm{M}\right)\end{array}$ & $\begin{array}{c}\text { RMSEP } \\
\left(\mathrm{x} 10^{-5} \mathrm{M}\right)\end{array}$ & $\mathrm{R}_{\mathrm{c}}{ }^{2}$ & $\mathrm{R}_{\mathrm{P}}{ }^{2}$ & Electrode & Analyte \\
\hline BAS_MSC_MC & 2 & 1.0312 & 1.6110 & 0.7615 & 0.4737 & AuAT/CNT/AuBT & Cys \\
SMOTH_MC_15PT & 1 & 2.3585 & 1.3264 & 0.0171 & 0.8507 & AuAT/CNT/AuBT & Cys \\
BAS_MC & 3 & 1.2071 & 1.2635 & 0.6732 & 0.6017 & AuAT/CNT/AuBT & Cys \\
GA+BAS_MC & 7 & 0.1126 & 0.4919 & 0.9971 & 0.9542 & AuAT/CNT/AuBT & Cys \\
MSC_MC & 5 & 0.2921 & 1.0597 & 0.9808 & 0.7280 & AuAT/CNT/AuBT & GSH \\
SMOTH_MC_15PT & 3 & 1.1075 & 0.6371 & 0.7249 & 0.9315 & AuAT/CNT/AuBT & GSH \\
BAS_MC & 5 & 0.2801 & 1.1366 & 0.9823 & 0.8907 & AuAT/CNT/AuBT & GSH \\
GA+BAS_MC & 6 & 0.1089 & 0.5047 & 0.9973 & 0.9429 & AuAT/CNT/AuBT & GSH \\
SMOTH_MC_15PT & 1 & 2.0443 & 1.9675 & 0.1805 & 0.6590 & AuAT/CNT/AuBT & hCys \\
SMOTH-MC & 5 & 0.4120 & 1.5526 & 0.9700 & 0.0092 & AuAT/CNT/AuBT & hCys \\
iPLS+MC & 7 & 02569 & 1.6732 & 0.9883 & 0.0859 & AuAT/CNT/AuBT & hCys \\
GA+iPLS-MC & 5 & 0.9340 & 0.4918 & 0.8289 & 0.9589 & AuAT/AuBT & hCys \\
\hline
\end{tabular}

Fig. 6. Plot of predicted concentrations $v s$. reference values for a) Cys. b) GSH. c) hCys. Calibration samples $(\bullet)$, external validation samples $(\boldsymbol{\nabla})$.
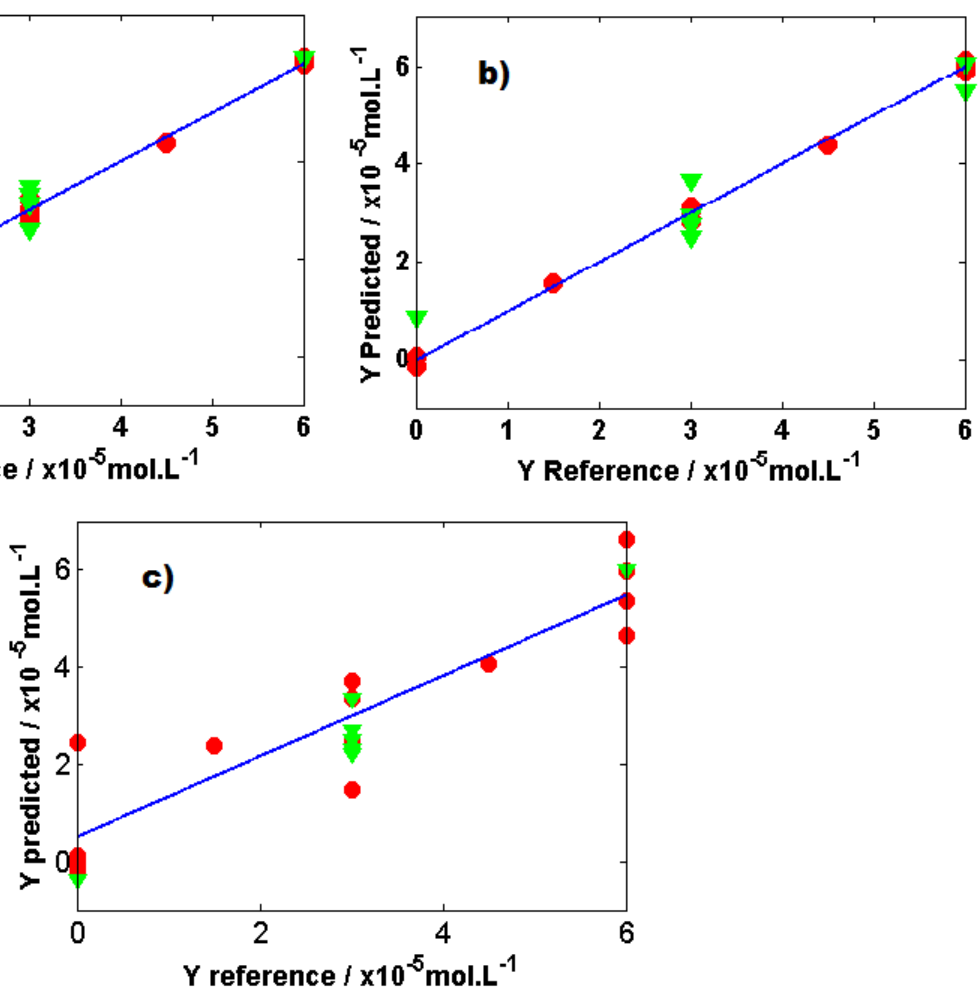


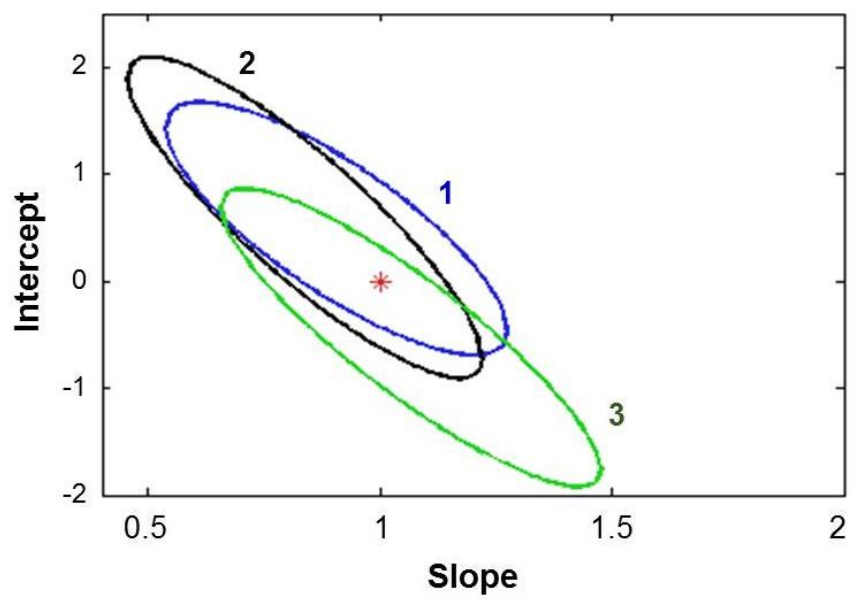

Fig. 7. Elliptical Joint Confidence Regions (at 95\% confidence level) for the slope and intercept of the regression of the predicted concentration versus reference value for Cys (1), GSH (2) and hCys (3).

\section{Conclusions}

In this work, a simple, fast and low cost methodology has been tested for the simultaneous analysis of cysteine, glutathione and homocysteine with no need of previous separation. It is based on the use of three different screenprinted electrodes operating together as a voltammetric electronic tongue. Although the signals produced by the three devices do not show well-defined peaks and in some cases they can be affected by capacitive background and noise, they proved to be enough sensitive, selective and different with each other as to allow a reasonable quantification of all three aminothiols by PLS calibration assisted by some preprocess and variables selection algorithms.

This preliminary test has been carried out with synthetic mixtures only, but the results here reported allow us to be optimistic about the future application of a similar, improved, methodology to the analysis of real biological samples without previous separation. The fundamentals of the 'electronic tongue' strategy have been roughly settled in this work. Now it is time to analyze more involved samples and add, when necessary, complementary screenprinted devices and alternative excitation signals (e.g. differential pulse or square wave scans) to increase the selectivity and robustness of the system.

\section{Acknowledgments}

This work is supported by the Ministry of Science and Innovation of Spain (Project CTQ2012-32863) and the Generalitat of Catalonia (Project 2014SGR269). R. H. P.
Pedroza also thanks the National Council for Scientific and Technological Development (CnPQ) of Brazil for the support and opportunity.

\section{References}

[1] P. C. Jocelyn, Biochemistry of the SH Group, Academic Press, London, 1972.

[2] H. Refsum, P. M. Ueland, O. Nygård, S. E. Vollset, Annu. Rev. Med. 1998, 49, 31-62.

[3] D. M. Townsend, K. D. Tew, H. Tapiero, Biomed. Pharmacother. 2004, 58, 47-55.

[4] G. Noctor, C. H. Foyer, Annu. Rev. Plant Physiol. Plant Mol. Biol. 1998, 49, 249-279.

[5] X. Chen, Y. Zhou, X.Peng, J. Yoon, Chem. Soc. Rev. 2010, 39, 2120-2135.

[6] P. C. White, N. S. Lawrence, J. Davis, R. G. Compton, Electroanalysis 2002, 14, 89-98.

[7] J. Kruusma, A. M. Benham, J. A. G. Williams, R. Kataky, Analyst 2006, 131, 459-473.

[8] J. C. Harfield, C. Batchelor-McAuley, R. G. Compton, Analyst 2012, 137, 2285-2296.

[9] I. R. Miller, J. Teva, J. Electroanal. Chem. 1972, 36, 157166.

[10] R. R. Moore, C. E. Banks, R. G. Compton, Analyst 2004, 129, 755-758.

[11] C. Terashima, T. N. Rao, B. V. Sarada, Y. Kubota, A. Fujishima, Anal. Chem. 2003, 75, 1564-1572.

[12] A. Sarakbi, Z. Aydogmus, A. Dago, D. Mertens, J. Y. Dewert, J. M. Kauffmann, Anal. Chim. Acta 2013, 786, 2228.

[13] N. Karadas-Bakirhan, A. Sarakbi, M. Vandeput, S. A. Ozkan, J. M. Kauffmann, Anal. Chem. 2015, 87, 67306735.

[14] N. W. H. Adams, J. R. Kramer, Aquat. Geochem. 1999, 5, $1-11$.

[15] R. D. Thackrey, T. L. Riechel, J. Electroanal. Chem. 1988, 245, 131-143.

[16] X. Qi, R. P. Baldwin, J. Electrochem. Soc. 1996, 143, 1283-1287.

[17] X. Luo, A. Morrin, A. J. Killard, M. R. Smyth, Electroanalysis 2006, 18, 319-326.

[18] Y. Xian, Y. Zhou, H. Wang, L. Zhou, F. Liu, L. Jin, J. Chromatogr. B 2005, 817, 239-246.

[19] L. Agüí, C. Peña-Farfal, P. Yánez-Sedeño, J. M. Pingarrón, Talanta 2007, 74, 412-420.

[20] S. Buratti, B. Brunetti, S. Mannino, Talanta 2008, 76, 454457.

[21] O. Domínguez Renedo, M. A. Alonso-Lomillo, M. J. Arcos Martínez, Talanta 2007, 73, 202-219.

[22] J. P. Metters, R. O. Kadara, C. E. Banks, Analyst 2011, 136, 1067-1076. 
[23] M. Li, Y. T. Li, D. W. Li, Y. T. Long, Anal. Chim. Acta 2012, 734, 31-44.

[24] N. Serrano, A. Alberich, J. M. Díaz-Cruz, C. Ariño, M. Esteban, Trends Anal. Chem. 2013, 46, 15-29.

[25] K. Duarte, C. I. L. Justino, A. C. Freitas, A. M. P. Gomes, A. C. Duarte, T. A. P. Rocha-Santos, Trends Anal. Chem. 2015, 64, 183-190.

[26] W. Y. Su, S. H. Cheng, Electrochem. Commun. 2008, 10, 899-902.

[27] C. Y. Liao, J. M. Zen, Sens. Actuators B 2008, 129, 896902.

[28] A. V. Eremenko, E. A. Dontsova, A. P. Nazarov, E. G. Evtushenko, S. V. Amitonov, S. V. Savilov, L. F. Martynova, V. V. Lunin, I. N. Kurochkin, Electroanalysis 2012, 24, 573-580.

[29] P. T. Lee, D. Lowinsohn, R. G. Compton, Sensors 2014, 14, 10395-10411.

[30] S. Cavanillas, N. Serrano, J. M. Díaz-Cruz, C. Ariño, M. Esteban, Electroanalysis 2014, 26, 581-587.

[31] M. Esteban, C. Ariño, J. M. Díaz-Cruz, Trends Anal. Chem. 2006, 25, 86-92.

[32] Y. Ni, Y. Wang, S. Kokot, Talanta, 2006, 69, 216-225.

[33] A. Rouhollahi, R. Rajabzadeh, J. Ghasemi, Microchim. Acta 2007, 157, 139-147.

[34] G. D. Pierini, N. E. Llamas, W. D. Fragoso, S. G. Lemos, M. S. Di Nezio, M. E. Centurión, Microchem. J. 2013, 106, 347-350.

[35] M. B. Gholivand, A. R. Jalalvand, H. C. Goicoechea, T. Skov, Talanta 2014, 119, 553-563.

[36] M. Behpour, S. Masoum, A. Lalifar, A. Khoobi, Sensors Act. B: Chemical 2015, 214, 10-19.

[37] R. Gusmão, C. Ariño, J. M. Díaz-Cruz, M. Esteban, Anal. Biochem. 2010, 406, 61-69.

[38] P. Ciosek, W. Wróblewskia, Analyst, 2007, 132, 963-978.

[39] A. Gutés, F. Céspedes, M. del Valle, Anal. Chim. Acta 2007, 600, 90-96.

[40] F. Winquist, Microchim. Acta 2008, 163, 3-10.

[41] M. del Valle, Electroanalysis 2010, 22, 1539-1555.

[42] Y. Tahara, IEEE Sensors Journal 2013, 13, 3001-3011.

[43] Matlab, version R2008b ed., Mathworks Inc.: Natick, MA, USA, 2008.

[44] P. Fanjul-Bolado, P. J. Lamas-Ardisana, D. HernándezSantos, A. Costa-García, Anal. Chim. Acta 2009, 638, 133138.

[45] PLS-toolbox version 7.8.2 (Eigenvector Research Inc., Wenatchee, USA).

[46] M. B. Gholivand, A. R. Jalalvand, H. C. Goicoechea, R. Gargallo, T. Skov, G. Paimard, Talanta 2015, 131, 26-37.

[47] R. E. L. Abreu, J. E. M. Paz, A. C. Silva, M. J. C. Pontes, S. G. Lemos, Fuel 2015, 156, 20-25.

[48] R. H. P. Pedroza, J. T. N. Nicácio, B. Souza dos Santos, Anal. Letters 2013, 46, 1145-1154.
[49] R. W. Kennard, L. A. Stone, Technometrics 1969, 11, 137148

[50] M. Vosough, S.N. Eshlaghi, R. Zadmard, Spectrochim. Acta 2015, 136, 618-624. 